\title{
Non-trivial, static, geodesically complete, vacuum space-times with a negative cosmological constant
}

\author{
Michael T. Anderson* \\ Department of Mathematics \\ S.U.N.Y. at Stony Brook \\ Stony Brook, N.Y. 11794-3651 \\ Piotr T. Chruściel ${ }^{\dagger}$ \\ Albert Einstein Institute ${ }^{\ddagger}$ \\ D-14476 Golm, Germany \\ Erwann Delay ${ }^{\S}$ \\ Département de mathématiques \\ Faculté des Sciences \\ Parc de Grandmont \\ F37200 Tours, France
}

\begin{abstract}
We construct a large class of new singularity-free static Lorentzian fourdimensional solutions of the vacuum Einstein equations with a negative cosmological constant. The new families of metrics contain space-times with, or without, black hole regions. Two uniqueness results are also established.
\end{abstract}

\section{Introduction}

It is part of the folklore expectations in general relativity that the following statements hold for solutions of Einstein's equations, with or without a cosmological constant:

- Static non-singular solutions possess at least three linearly independent local Killing vector fields near each point.

${ }^{*}$ Partially supported by NSF Grant DMS 0072591; email anderson@math.sunysb.edu

${ }^{\dagger}$ Partially supported by a Polish Research Committee grant; email piotr@gargan.math. univ-tours.fr

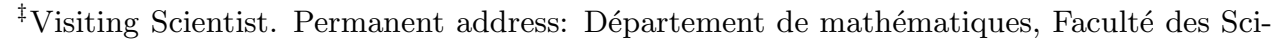
ences, Parc de Grandmont, F37200 Tours, France

${ }^{\S}$ Partially supported by the ACI program of the French Ministry of Research; email delayd gargan.math.univ-tours.fr 
- Stationary non-singular solutions possess at least two linearly independent local Killing vector fields near each point.

By local Killing vector fields we mean those solutions of the Killing equations which are defined in a neighborhood of some point, and which do not necessarily extend to global solutions. There is a wide body of evidence that these statements are correct when the cosmological constant vanishes of or is positive (see [2, 1, 11, 10, 33, 27] and references therein), and some very partial results indicating that this could perhaps be true when the cosmological constant $\Lambda$ is negative 12, 13, 17, 16, 9]. The object of this paper is to show that such rigidity is false in this last situation. More precisely, for $\Lambda<0$ there exist 4-dimensional strictly globally static $\beta^{\beta}$ solutions $(\mathscr{M}, \mathfrak{g})$ of the vacuum Einstein equations with the following properties:

1. $(\mathscr{M}, \mathfrak{g})$ is diffeomorphic to $\mathbb{R} \times \Sigma$, for some 3 -dimensional spacelike Cauchy surface $\Sigma$, with the $\mathbb{R}$ factor corresponding to the action of the isometry group.

2. $\left(\Sigma, g_{\Sigma}\right)$, where $g_{\Sigma}$ is the metric induced by $\mathfrak{g}$ on $\Sigma$, is a complete Riemannian manifold.

3. $(\mathscr{M}, \mathfrak{g})$ is geodesically complete.

4. All invariants of $\mathfrak{g}$ which are algebraically constructed using the curvature tensor and its derivatives up to any finite order are uniformly bounded on $\mathscr{M}$.

5. $(\mathscr{M}, \mathfrak{g})$ admits a globally hyperbolic (in the sense of manifolds with boundary $\mathbb{f}^{\mathbb{I}}$ smooth conformal completion with a timelike $\mathscr{I}$.

6. $\left(\Sigma, g_{\Sigma}\right)$ has a $C^{\infty}$ conformal compactification.

7. The connected component of the group of isometries of $(\mathscr{M}, \mathfrak{g})$ is exactly $\mathbb{R}$, with an associated Killing vector $X$ being timelike throughout $\mathscr{M}$.

8. There exist no local solutions of the Killing equation other than the (globally defined) timelike Killing vector field $X$.

\footnotetext{
${ }^{1}$ An example is given by rotational Killing vector fields on a torus, which exist in a neighborhood of each point, but which do not extend to globally defined vector fields. The restriction to local Killing vector fields is necessary in the statements above: the four dimensional higher genus Kottler black holes have four locally defined Killing vector fields in a some neighborhood of each point, but only one which is globally defined.

${ }^{2}$ In the $\Lambda=0$ case the only exception known to us is provided by the Myers - NicolaiKorotkin metrics 30,25] which are, however, not asymptotically flat in the sense which one usually uses in the context of black hole space-times.

${ }^{3}$ We shall say that a space-time is strictly globally static if it contains a (strictly) timelike Killing vector field which is orthogonal to the level sets of a globally defined time function.

${ }^{4}$ We say that a space-time with boundary is globally hyperbolic if it contains a Cauchy surface; the latter are defined as hypersurfaces which are intersected by every inextendible causal curve precisely once.
} 
An example of a manifold satisfying points 1 -6 above is of course anti-de Sitter space-time. Clearly the anti-de Sitter solution does not satisfy points 7 and 8 .

One of the main results of this paper is a general existence theorem producing a large class of space-times satisfying $1-8$, with prescribed data at conformal infinity.

Theorem 1.1 Any $C^{\infty}$ strictly globally static Lorentz metric $\gamma$ on $\mathbb{R} \times S^{2}$, i.e.

$$
\gamma=-\alpha^{2} d t^{2}+g_{S^{2}}
$$

with $\alpha>0$ and of non-negative scalar curvature on $\mathbb{R} \times S^{2}$, is the conformal infinity of a complete, strictly globally static vacuum metric on $\mathbb{R} \times \mathbb{R}^{3}=\mathbb{R}^{4}$, with cosmological constant $\Lambda=-6$.

Generic space-times in this result satisfy points 1-8 (if $\gamma$ has another Killing vector, then $g$ also will; in particular the construction also yields non-trivial static-axially symmetric vacuum metrics). A related, but weaker, existence result is proved for space-times with $\Lambda<0$ and with conformal infinity of the topological form $\mathbb{R} \times T^{2}$.

An existence result of this kind is also proved for space-times with black holes of any given genus at the horizon and at conformal infinity, (although again this result is not as general as Theorem 1.1 in terms of the prescribed boundary data at infinity, see the discussion before (3.13)). In the black hole context, Points 1-8 remain correct, with the following obvious variations: Point 1 remains correct if $\mathscr{M}$ is replaced by its domain of outer communications. Point 2 remains correct if completeness is understood in the sense of manifolds with (minimal) boundary. Finally, completeness holds for geodesics starting in the domain of outer communication except perhaps for those which cross the future or past event horizons. The remaining points remain correct without modifications.

Throughout this work we restrict attention to dimension $n=4$. Similar but weaker results can be obtained in higher dimensions; this will be discussed elsewhere. Let us simply mention that arguments used below provide, e.g., existence of vacuum $(n+1)$-dimensional strictly globally static metrics, with the associated boundary metrics lying in a neighborhood of $-d t^{2}+g_{+1}$, where $g_{+1}$ is the round unit metric on $S^{n-1}$ (this is the boundary metric arising from the $(n+1)$-dimensional anti-de Sitter metric). Further, using Kaluza-Klein reduction one can in particular obtain space-times as above, with or without black hole regions, which satisfy the Einstein-Yang-Mills-dilaton field equations; solutions belonging to this family have been numerically constructed in [8, 34]; see also [6].

It is clear that there should exist stationary and not static solutions as above, which can be constructed by solving an asymptotic Dirichlet problem for the stationary Einstein equations in a conformally compactifiable setting. We are planning to study this question in a near future.

This paper is organised as follows: In Section 2 we review those results of [3, 4] which are needed for our work here, and we give conditions under which hypersurface-orthogonality descends from the conformal boundary to the interior. In Section 3 we prove our existence and uniqueness results. In Section 4 we justify the claims 118 made above. 


\section{Isometry Extension}

Let $M$ be an oriented 4-manifold with compact boundary $\partial M$. In this section, we work exclusively with Riemannian metrics. The results obtained here will be applied to Lorentzian metrics in Section 3.

Let $E=E_{A H}^{m, \alpha}$ denote the space of conformally compact or asymptotically hyperbolic (AH) Einstein metrics on $M$; thus $g \in E_{A H}^{m, \alpha}$ if $g$ is an Einstein metric on $M$,

$$
R i c_{g}=-3 g
$$

and $g$ has a $C^{m, \alpha}$ conformal compactification, $m \geq 2, \alpha>0$. By this we mean that the metric $\rho^{2} g$ extends by continuity to a $C^{m, \alpha}$ Riemannian metric on $\bar{M}$, where $\rho$ is any function which vanishes precisely on $\partial M$, and has nowhere vanishing gradient there. Let $\mathcal{E}=\mathcal{E}_{A H}^{m, \alpha}$ be the moduli space of such Einstein metrics, i.e. $\mathcal{E}_{A H}^{m, \alpha}=E_{A H}^{m, \alpha} / \mathcal{D}_{1}$, where $\mathcal{D}_{1}$ is the group of $C^{m+1, \alpha}$ diffeomorphisms of $\bar{M}$ which restrict to the identity on $\partial M$.

Let $\mathcal{C}=\mathcal{C}^{m, \alpha}$ be the space of pointwise conformal classes of $C^{m, \alpha}$ metrics on $\partial M$. One then has a natural boundary map

$$
\Pi: \mathcal{E} \rightarrow \mathcal{C}, \Pi[g]=[\gamma]
$$

which associates to each class $[g]$ its conformal infinity $[\gamma]$ on $\partial M$.

From $\llbracket$, Thm. A], $\mathcal{E}$ is either empty, or an infinite dimensional Banach manifold, and $\Pi$ is a smooth Fredholm map of Fredholm index 0. Thus, if non-empty, Im $\Pi$ is a "variety" of finite codimension in $\mathcal{C}$. This result holds for any $m \geq 2$, in particular for $m=\infty$ or for $m=\omega$, i.e. real-analytic.

For $g \in \mathcal{E}$, let $K=K_{g}$ be the $L^{2}$ kernel of $D \Pi$, i.e. the space of symmetric bilinear forms $h$ on $(M, g)$ which are in $L^{2}(M, g)$ and satisfy $D \Pi(h)=0$. At any $g \in \mathcal{E}$ for which $K_{g}=0$, the map $D \Pi$ is an isomorphism and hence $\Pi(\mathcal{E})$ contains an open neighborhood of $\gamma=\Pi(g)$ in $\mathcal{C}$, cf. [19, 7, 28]. Thus, if $\mathcal{E}$ contains a regular point of $\Pi$, then $\operatorname{Im} \Pi \subset \mathcal{C}$ is a variety of codimension 0 in $\mathcal{C}$. It is natural to conjecture that $\mathcal{E}$ always contains such a regular point, and in fact such regular points are dense in $\mathcal{E}$, although these issues are currently unresolved in general.

The starting point of the work in this section is the following result औ, Thm. 3.2]:日

Proposition 2.1 Let $g$ be an AH Einstein metric on a 4-manifold $M, g \in$ $\mathcal{E}_{A H}^{m, \alpha}, m \geq 4$, with conformal infinity $[\gamma]$. Then any connected group $G$ of effective conformal isometries of $(\partial M, \gamma)$ extends to a connected group $G$ of effective isometries of $(M, g)$.

In particular, if $(\partial M, \gamma)=\left(S^{3}, \gamma_{0}\right)$ is the round metric on $S^{3}$, then any $\mathrm{AH}$ Einstein filling metric has an effective isometric $S O(3,1)$ action. The only such

\footnotetext{
${ }^{5}$ It is unfortunate that Proposition 2.1 does not necessarily hold for isometries which do not lie in the connected component of the group, as such a property would have considerably simplified some of the arguments below.
} 
metric is the hyperbolic metric $g_{-1}$ on the 4 -ball $B^{4}$. In all other cases, by the well-known Obata Theorem, (the conformal isometry group is equivalent to the isometry group unless $\gamma$ is the round metric on $S^{3}$ ), one may assume that $G$ is a group of isometries of $(\partial M, \gamma)$.

Thus, let $G=S^{1}$, and suppose that we have an isometric $S^{1}$ action on $(\partial M, \gamma)$. It follows that this action extends to an isometric $S^{1}$ action on any AH Einstein filling metric $(M, g)$, with associated Killing field $K$.

For an AH Einstein metric with an effective $S^{1}$ action on $M$, there are in general three types of $S^{1}$ orbits:

- principal orbits, on which the action is free;

- exceptional orbits, on which the action has a non-trivial but finite isotropy group;

- fixed points.

The fixed point set $F$ consists of a finite collection of smooth surfaces $B=$ $\cup B_{i}$ embedded in $M$, and a finite number of isolated points $N=\cup n_{j}$; these are called bolts and nuts respectively in the terminology of Gibbons-Hawking [18]. In general, the bolts might intersect infinity, i.e. $\partial M$, and so be complete open surfaces in $M$. This will be the case if the Killing field $K$ vanishes on circles in $\partial M$. For simplicity, we rule out zeros of $K$ at $\partial M$, and in fact we will only consider free $S^{1}$ actions on $\partial M$.

Let $\Sigma=M / S^{1}$ be the orbit space of the $S^{1}$ action, with $\pi: M \rightarrow \Sigma$ the projection to the orbit space. By [15, 14], $\Sigma$ is topologically a 3 -manifold with boundary, with $\partial \Sigma=B$. Of course the nuts correspond to isolated points in $\Sigma$. Note that $\Sigma$ also has a boundary at infinity $\partial_{\infty} \Sigma$ corresponding to the quotient $\partial M / S^{1}$. Let $E$ denote the exceptional set, i.e. the union of the exceptional orbits in $M$. Then the quotient $\Sigma_{E}=\pi(E)$ consists of a finite number of smooth embedded curves $\sigma_{k}$ in $\Sigma$, along which the isotropy is constant. Each curve $\sigma_{k}$ is either a closed curve in the interior $\stackrel{\circ}{\Sigma}$, or is an arc with distinct endpoints $\partial \sigma_{k} \subset \partial \Sigma$. Let $P \subset M$ denote the union of the principal orbits of the $S^{1}$ action. Then $P$ is open and dense in $M$ and $\Sigma_{P}=\pi(P)$ is open and dense in $\Sigma$.

The metric $g$ on $M$ induces a metric $g_{\Sigma}$ on the orbit space, for which the projection $\pi$ is a Riemannian submersion. It follows that $g$ may be written in the form

$$
g=u^{2}(d \phi+\theta)^{2}+\pi^{*} g_{\Sigma},
$$

where $\theta$ is a connection 1-form, $u$ is the length of the Killing field $K$ and $K=\partial / \partial \phi$. The parameter $\phi$ parameterises a circle $S^{1}$.

The nuts and bolts in $M$ are exactly the locus where the Killing field $K$ vanishes, i.e. the 0 -set of the function $u$. It is well-known that sets of fixed points are totally geodesic, cf. [24], and hence the boundary $\partial \Sigma$ is totally geodesic in

\footnotetext{
${ }^{6}$ The fact that the hyperbolic metric is the only Einstein metric on a spin 4-manifold with conformal infinity $\left(S^{3}, \gamma_{0}\right)$ has already been established by rather different methods in [0].
} 
$\Sigma$. The same argument shows that the exceptional curves $\gamma_{k}$ are geodesics in $\left(\Sigma, g_{\Sigma}\right)$. The metric $g_{\Sigma}$ is not smooth along each $\gamma_{k}$, but has normal cone singularities, i.e. metrically the normal disc to $\gamma_{k}$ at a point of $\gamma_{k}$ is of the form $\mathbb{R}^{2} / \mathbb{Z}_{k^{\prime}}$, where the cyclic group $\mathbb{Z}_{k^{\prime}}$ acts in the usual way by rotation on $\mathbb{R}^{2}$; here of course the order $k^{\prime}$ might depend on $k$.

We will use the following terminology. The $S^{1}$ action on $(M, g)$ is strictly globally static if $(M, g)$ is globally a warped product of the form

$$
M=S^{1} \times \Sigma, g=u^{2} d \phi^{2}+\pi^{*} g_{\Sigma},
$$

where $u: \Sigma \rightarrow \mathbb{R}$ is strictly positive and $g_{\Sigma}$ is a complete metric on $\Sigma, \partial \Sigma=\emptyset$. In this case, the $S^{1}$ action is just given by rotations in the $S^{1}$ factor; there are no nuts, bolts or exceptional orbits. The $S^{1}$ action is globally static if (2.3) holds with $u=0$ somewhere. In this case, the locus $\{u=0\}$ is given by bolts - there are no nuts or exceptional orbits. Next, the $S^{1}$ action is topologically static if the $S^{1}$ bundle $S^{1} \rightarrow P \rightarrow \Sigma_{P}$ is a trivial bundle, i.e. it admits a section. This is equivalent to the existence of a cross-section of the $S^{1}$ fibration $P \cup E \rightarrow \Sigma_{P \cup E}$. \ Finally, we define the $S^{1}$ action to be locally static if every point of $(M, g)$ has a neighborhood isometric to a neighborhood of a point with metric of the form (2.3); this is equivalent to the usual notion of static in terms of the existence of a hypersurface orthogonal Killing field. It is easy to see that a locally static action has no nuts. [

We shall use an obvious equivalent of the above for an $\mathbb{R}$ action by isometries on a Lorentzian manifold $(\mathscr{M}, \mathfrak{g})$, with the further restriction that the associated Killing vector field be timelike almost everywhere.

The main result of this section is the following:

Theorem 2.2 Let $(M, g)$ be as above, with $g \in \mathcal{E}^{m, \alpha}, m \geq 4$. Suppose the free $S^{1}$ action at conformal infinity $(\partial M, \gamma)$ is strictly globally static, i.e.

$$
\partial M=S^{1} \times V
$$

and the $S^{1}$ action on $(M, g)$ is topologically static.

Then the $S^{1}$ action on $(M, g)$ is locally static, i.e. $(M, g)$ is locally of the form (2.3), (with $\{u=0\} \neq \emptyset$ possibly).

REMARK 2.3 The result remains true if the hypothesis of topological stability is replaced by the requirements that a) $\stackrel{\circ}{\Sigma}^{\circ}$ is simply connected and b) $\{u=0\}$ is connected, if not empty. This can be established by an analysis of the equation satisfied by the twist potential.

PRoof: The proof is rather long, and will be broken into two steps.

\section{Step I.}

\footnotetext{
${ }^{7} S$ will be called a cross-section of a fibration if $S$ meets every fiber at least once, with the intersection being transverse.

${ }^{8}$ For a nut $n \in M$, the geodesic $\varepsilon$-sphere $S_{n}(\varepsilon)$ is invariant under the action, which is free. But $S_{n}(\varepsilon)$ is topologically $S^{3}$ for sufficiently small $\varepsilon$, which admits no free (even topologically) static $S^{1}$ actions.
} 
In this step, we set up the basic equations and obtain estimates on the behavior near infinity.

Let $\omega$ be the twist 1-form, given by

$$
\omega=\frac{1}{2} u^{3} *_{\Sigma} d \theta=\frac{1}{2} *_{M}(\kappa \wedge d \kappa),
$$

where $\kappa=u^{2}(d \phi+\theta)=g(K, \cdot)$ is the 1 -form dual to the Killing field $K$, cf. [26, (16.7), (16.32)]. Here, the first $*$ operator $*_{\Sigma}$ is on $\left(\Sigma, g_{\Sigma}\right)$, while the second is on $(M, g)$. The twist form is horizontal, i.e. $\omega(K)=0$ and is invariant under the $S^{1}$ action, so descends to a 1 -form on $\Sigma$.

The condition that the $S^{1}$ action on $(M, g)$ is locally static is equivalent to the condition that

$$
\omega \equiv 0 \text {. }
$$

The Einstein equations imply that the 1 -form $\omega$ is closed,

$$
d \omega=0
$$

It follows from (2.5) and (2.6) that, on the orbit space $\Sigma$, one has

$$
\operatorname{div}\left(u^{-3} \omega\right)=0
$$

Hence, the divergence theorem applied to any bounded smooth domain $U$ in $\Sigma$ implies

$$
\int_{\partial U} u^{-3} \omega(\nu)=0
$$

where $\nu$ is the outward unit normal. In particular, choosing $U$ to be a fixed connected neighborhood of any bolt $B_{i}$ and replacing $U$ by $U \backslash B_{i}(\varepsilon)$, where $B_{i}(\varepsilon)$ is the $\varepsilon$-tubular neighborhood of $B_{i}$, it follows that

$$
\int_{B_{i}} u^{-3} \omega(\nu) d A=\kappa_{i}
$$

is well-defined, where $\nu$ is a unit normal to $B_{i}$ in $\Sigma$. Similarly, if $S_{j}(\varepsilon)$ is the $\varepsilon$-sphere about the nut $n_{j}$, then the limit

$$
\lim _{\varepsilon \rightarrow 0} \int_{S_{j}(\varepsilon)} u^{-3} \omega(\nu) d A=\kappa_{j},
$$

exists, (and is finite). These integrals give the nut charge of each bolt or nut, in the terminology of [18, §5]. Note also that since $\omega \equiv 0$ on any bolt or nut, to leading order, $\omega(\nu) \sim \pm|\omega|$ on $S_{j}(\varepsilon)$, where $S_{j}(\varepsilon)$ is the boundary of the $\varepsilon$-tubular neighborhood of the bolt or nut.

Next we turn to the asymptotic behavior of the metric $g$. Asymptotically, the metric $g=g_{\Sigma}$ on $\Sigma$ has the form

$$
g=r^{-2} d r^{2}+r^{2} \gamma+h,
$$


where $\gamma$ is the boundary metric on $\partial M$, and $\|h\|=O\left(r^{-2}\right)$, w.r.t the metric $g$, cf. [4, §2]. The function $u$ satisfies $u \sim r$ for large $r$, i.e. the ratio $u / r$ is bounded away from 0 and $\infty$. From (2.10), observe that

$$
|\nabla r|=|d r|=r
$$

Let $\bar{g}=\rho^{2} g$ be a smooth, (at least $C^{4, \alpha}$ ), compactification of $g$. Thus, $\rho \sim r^{-1}, u \rho \sim 1$ and

$$
\bar{g}=\rho^{2} u^{2}(d \phi+\theta)^{2}+\rho^{2} g_{\Sigma} .
$$

To be concrete, choose $\rho$ to be the geodesic defining function determined by a choice of boundary metric $\gamma \in[\gamma]$. Since the Killing field preserves the class of geodesics orthogonal to $\partial M, \rho$ is invariant under the $S^{1}$ action, and so descends to a defining function for $\partial_{\infty} \Sigma$. In particular, $d \phi$ and $\theta$ are the same for $g$ and $\bar{g}$, while the norm of the Killing field w.r.t. $\bar{g}$ is $\rho u$.

Let $\bar{\omega}$ be the twist form, defined as in (2.5), but w.r.t. the $\bar{g}$ metric. Then $\bar{\omega}$ extends smoothly to $(\bar{M}, \bar{g})$, since the compactification is smooth. A direct computation from (2.5) shows that, near $\partial M$,

$$
2 \bar{\omega}=(u \rho)^{4} \bar{*}_{M}[(d \phi+\theta) \wedge d \theta]=(u \rho)^{3} \bar{*}_{\Sigma} d \theta=2(u \rho)^{3} \rho^{-1} u^{-3} \omega .
$$

Here, we use the fact that $\|d \phi\|_{\bar{g}}=(u \rho)^{-1}$ and $\bar{*}_{\Sigma}=\rho^{-1} *_{\Sigma}$ on 2 -forms. Thus

$$
\bar{\omega}=\rho^{2} \omega .
$$

In particular, one has

$$
|\bar{\omega}|_{\bar{g}}=\rho|\omega|_{g}
$$

Since the action on the boundary is static, it follows that $\bar{\omega}=0$ on $\partial M$. Since the compactification is smooth, $|\bar{\omega}|_{\bar{g}}=O(\rho)$, and hence (2.14) gives

$$
|\omega|=O(1)
$$

We need to improve this estimate by order 1 . This is done in the following result.

Lemma 2.4 The twist form $\omega=\rho^{-2} \bar{\omega}$ extends smoothly to $\partial M$, with

$$
|\omega|=O(\rho) .
$$

Proof: By (2.13) and (2.14), it suffices to show that $\bar{\omega}=O\left(\rho^{2}\right)$ near $\partial M$. Of course both $d \bar{\omega}$ and $\bar{\omega}$ are smooth up to $\partial M$.

By (2.13), we have

$$
d \bar{\omega}=d\left(\ln \rho^{2}\right) \wedge \bar{\omega}
$$

We claim first that if the action is static at $\partial M$, then

$$
d \bar{\omega}=0 \text { at } \partial M .
$$

To see this, by standard formulas, cf. [21, p.10] for example, one has in general

$$
d \bar{\omega}=*(K \wedge \bar{R} i c(K)),
$$


where $K$ is the Killing field w.r.t. $\bar{g}$, (and $K$ is identified with the 1 -form $\kappa$ by $\bar{g})$. Thus, the vanishing - or not - of $d \bar{\omega}$ is governed by $\langle\bar{R} i c(K), X\rangle$, where $X$ is orthogonal to $K$. However, using the formulas of [3, Lemma 1.2] for instance, the ambient Ricci curvature $\bar{R} i c$ at $\partial M$ is determined by the intrinsic Ricci curvature of the boundary metric $\gamma$; in fact for $X, Y$ tangent to $\partial M$,

$$
\bar{R} i c(X, Y)=2 \operatorname{Ric}_{\gamma}(X, Y)-\frac{1}{4} R_{\gamma} \cdot \gamma(X, Y) .
$$

Now if the $S^{1}$ action on $\partial M$ is static, then it is well-known that $\operatorname{Ric}_{\gamma}(K, X)=0$, for any $X$ orthogonal to $K$ and tangent to $\partial M$. Similarly, if $X=\bar{\nabla} \rho$, then $\bar{R} i c(K, X)=0$, by [3, Lemma 1.2] again. This gives (2.17). It follows that $\rho^{-1} d \bar{\omega}$ extends smoothly to $\partial M$.

By (2.16), this means that $\rho^{-2} \bar{\omega}(X)$ extends smoothly up to $\partial M$, for any smooth vector field $X$ orthogonal to $\bar{\nabla} \rho$. Thus, it remains to understand the behavior of $\bar{\omega}(\bar{\nabla} \rho)$. By $(2.6)$, one has

$$
\bar{\omega}(\bar{\nabla} \rho)=\frac{1}{2} \rho u d \kappa\left(T_{1}, T_{2}\right)=-\frac{1}{4} \rho u \bar{g}\left(K,\left[T_{1}, T_{2}\right]\right),
$$

where $K /|K|, T_{1}$ and $T_{2}$ are $\bar{g}$-orthonormal vector fields orthogonal to $\bar{\nabla} \rho$. Hence, at $\partial M, T_{1}$ and $T_{2}$ are tangent to the surface $V$ in (2.4). For further calculations it will be convenient to assume that $\bar{\nabla}_{T_{i}} T_{j}=0$ at the given (arbitrary) point in $V$. This is compatible with the requirement of tangency of the $T_{i}$ 's to $V$ because $\partial M$ is totally geodesic in $\bar{M}$, with respect to the $\bar{g}$ metric. Since the $S^{1}$ action is static at $\partial M$, one has

$$
\bar{\omega}(\bar{\nabla} \rho)=0 \text { at } \partial M,
$$

compare with (2.14). Thus, it suffices to prove that

$$
\bar{\nabla} \rho(\bar{\omega}(\bar{\nabla} \rho))=0 \text { at } \partial M .
$$

This is a direct computation: set $\beta=-\frac{1}{4} \rho u$ (which has a smooth limit on $\partial M$, still denoted by the same symbol) so that, using (2.18) one has

$$
\left.\bar{\nabla} \rho(\bar{\omega}(\bar{\nabla} \rho))\right|_{\partial M}=\beta \bar{g}\left(\bar{\nabla}_{\bar{\nabla} \rho} K,\left[T_{1}, T_{2}\right]\right)+\beta \bar{g}\left(K, \bar{\nabla}_{\bar{\nabla} \rho} \bar{\nabla}_{T_{1}} T_{2}-\bar{\nabla}_{\bar{\nabla} \rho} \bar{\nabla}_{T_{2}} T_{1}\right\rangle .
$$

For the first term, since $K$ is conformal Killing on $\partial M$, one has $\bar{g}\left(\bar{\nabla}_{\bar{\nabla} \rho} K,\left[T_{1}, T_{2}\right]\right)=-\bar{g}\left(\bar{\nabla}_{\left[T_{1}, T_{2}\right]} K, \bar{\nabla} \rho\right)+\lambda \bar{g}\left(\bar{\nabla} \rho,\left[T_{1}, T_{2}\right]\right)$, both of which vanish since the $2^{\text {nd }}$ fundamental form $A=0$ at $\partial M$.

To calculate the second term, observe first that for any horizontal field $T$ on $\partial M$, i.e. $T$ tangent to the $V$ factor in (2.4), $\bar{\nabla}_{\bar{\nabla} \rho} T$ is also horizontal. To see this, using the fact that $K$ is conformal Killing on $\partial M$, one has $\bar{g}\left(\bar{\nabla}_{\bar{\nabla} \rho} T, K\right)=$ $-\bar{g}\left(\bar{\nabla}_{\bar{\nabla} \rho} K, T\right)=\bar{g}\left(\bar{\nabla}_{T} K, \bar{\nabla} \rho\right)=0$, since again $A=0$. Now write

$\bar{g}\left(K, \bar{\nabla}_{\bar{\nabla} \rho} \bar{\nabla}_{T_{1}} T_{2}\right)=\bar{g}\left(K, \bar{\nabla}_{T_{1}} \bar{\nabla}_{\bar{\nabla} \rho} T_{2}\right)+\bar{g}\left(\bar{R}\left(\bar{\nabla} \rho, T_{1}\right) T_{2}, K\right)+\bar{g}\left(\bar{\nabla}_{\left[\bar{\nabla} \rho, T_{1}\right]} T_{2}, K\right\rangle$.

For the curvature term, the Gauss-Codazzi equations give $\bar{g}\left(\bar{R}\left(\bar{\nabla} \rho, T_{1}\right) T_{2}, K\right)=$ $d A\left(T_{1}, T_{2}, K\right)$, which vanishes since $A=0$ at $\partial M$. Further, again since $A=0$, 
$[\bar{\nabla} \rho, T]$ is tangent to $V$, and hence $\bar{g}\left(\bar{\nabla}_{\left[\bar{\nabla} \rho, T_{1}\right]} T_{2}, K\right)=0$ by 2.18). Finally, for the first term above, since $\bar{\nabla}_{\bar{\nabla} \rho} T_{2}$ is horizontal, so is $\bar{\nabla}_{T_{1}} \bar{\nabla}_{\bar{\nabla} \rho} T_{2}$, and hence the first term also vanishes.

This completes the proof. In fact, with further computation, it is not difficult to show that $\bar{\omega}(\bar{\nabla} \rho)$ vanishes to order 2 at $\partial M$.

\section{Step II.}

The remainder of the proof is an adaptation of a result of Heusler, 21, Thm.8.2], on stationary asymptotically flat Lorentz metrics to the current situation. The basic tool is the following Lemma, proved in the Lorentzian setting in [21, (8.4)]. The proof in the Riemannian setting is essentially the same, but for clarity we give a complete proof.

Lemma 2.5 Let $(M, g)$ be a Riemannian 4-manifold, with Killing field $K$, with $u^{2}=|K|^{2}$ and with twist 1 -form $\omega$. Then

$$
d\left(\omega \wedge \frac{\kappa}{u^{2}}\right)=d \omega \wedge \frac{\kappa}{u^{2}}-2 \frac{|\omega|^{2}}{u^{4}} * \kappa
$$

where $\kappa$ is the 1 -form dual to $K$ by the metric.

Proof: Clearly it suffices to show that

$$
\omega \wedge d\left(\frac{\kappa}{u^{2}}\right)=2 \frac{|\omega|^{2}}{u^{4}} * \kappa .
$$

To do this, we first claim that

$$
d\left(\frac{\kappa}{u^{2}}\right)=2 *\left(\kappa \wedge \frac{\omega}{u^{4}}\right) .
$$

To verify (2.21), a simple computation shows that for any 1-form $\alpha$ on $M^{4}$, one has

$$
\iota_{X}(* \alpha)=*(\alpha \wedge \xi),
$$

where $\xi$ is the 1 -form dual to the vector field $X$. Applying this to $X=K$ and $\alpha=\omega$ gives

$$
*(\kappa \wedge \omega)=-\iota_{K}(* \omega)=+\frac{1}{2} \iota_{K}(\kappa \wedge d \kappa),
$$

where the last inequality follows from the definition of $\omega$ and the fact that $*^{2}=$ -1 for 3 -forms on a 4-manifold. Interior multiplication is a skew-derivation, and so $\iota_{K}(\kappa \wedge d \kappa)=\iota_{K}(\kappa) d \kappa-\kappa \wedge \iota_{K}(d \kappa)$. One has $\iota_{K}(\kappa)=|K|^{2}=u^{2}$, while, since $\mathcal{L}_{K} \kappa=0, \iota_{K}(d \kappa)=-d \iota_{K}(\kappa)=-d u^{2}$. This gives

$$
2 *(\kappa \wedge \omega)=u^{2} d \kappa+\kappa \wedge d u^{2},
$$

or equivalently,

$$
u^{-2} d \kappa=-u^{-4} \kappa \wedge d u^{2}+2 u^{-4} *(\kappa \wedge \omega) .
$$

But $d\left(u^{-2} \kappa\right)=-u^{-4} d u^{2} \wedge \kappa+u^{-2} d \kappa$, which then gives (2.21). 
From (2.21), it follows that

$$
\omega \wedge d\left(\frac{\kappa}{u^{2}}\right)=2 u^{-4} \omega \wedge *(\kappa \wedge \omega)=-2 u^{-4} \omega \wedge \iota_{K}(* \omega),
$$

where the second equality follows from (2.22). But $\iota_{K}(\omega \wedge * \omega)=\iota_{K}(\omega) \wedge * \omega-$ $\omega \wedge \iota_{K}(* \omega)$. Since $\iota_{K} \omega=0,(\omega$ is horizontal while $K$ is vertical), one obtains

$$
\omega \wedge d\left(\frac{\kappa}{u^{2}}\right)=2 u^{-4} \iota_{K}(\omega \wedge * \omega)=2 u^{-4}|\omega|^{2} \iota_{K} d v o l_{M},
$$

which gives (2.20).

We apply this result to the AH Einstein manifold $(M, g)$. By $(2.6), d \omega=0$, and so (2.19) implies

$$
2 \frac{|\omega|^{2}}{u^{4}} * \kappa=-d\left(\omega \wedge \frac{\kappa}{u^{2}}\right) .
$$

Now by assumption, the $S^{1}$ action on $(M, g)$ is topologically static. Hence, there exists a cross-section $\iota: \Sigma_{P \cup E} \rightarrow(P \cup E) \subset M$, such that $\pi \circ \iota=i d$. Let $S=\operatorname{Im} \iota$. Thus $S$ is an embedded hypersurface in $M$, which may be perturbed slightly if necessary to obtain a smooth hypersurface $S$, transverse to the $S^{1}$ orbits. In addition, we may assume, without loss of generality, that $S$ is a 3 -manifold with boundary $\partial S$, (possibly empty). The boundary consists of bolts, together possibly with a finite collection of points corresponding to the nuts. (Hence, these are not actually in $\partial S$, but just distinguished points in $S$. The metric $\bar{g}_{\Sigma}$ might have a singularity there, which is sufficiently mild to be irrelevant for our purposes here.). Moreover, $S$ has a boundary at infinity,

$$
\partial_{\infty} S=V
$$

for $V$ as in (2.4), which may be assumed to be smooth, (in the given compactification $(\bar{M}, \bar{g}))$. Thus, $\left(S, g_{S}\right)$, where $g_{S}=\left.g\right|_{S}$ is a complete Riemannian 3 -manifold with boundary. The fact that $\partial_{\infty} S=V$ smoothly implies that we may assume the 3-manifold $S$ becomes orthogonal to the $S^{1}$ orbits on approach to $\partial M$, i.e.

$$
\langle K /|K|, e\rangle(x) \rightarrow 0, \text { as } x \rightarrow \partial M,
$$

where $e$ is any unit tangent vector to $S$ at $x$.

Now integrate (2.23) over $S$ and apply Stokes theorem to obtain

$$
2 \int_{S} \frac{|\omega|^{2}}{u^{4}} * \kappa=-\int_{\partial S} \omega \wedge \frac{\kappa}{u^{2}}
$$

On $S$, the 3 -form $* \kappa=\alpha|K| d v l_{S}$, where $\alpha$ is the sine of the angle between $T S$ and $K$, (at any given $x \in S$ ). Since $S$ is everywhere transverse to $K$, $\alpha>0$ everywhere, (or $<0$ everywhere, depending on the choice of orientation). Hence, the integrand on the left in 2.26) does not change sign.

The boundary integral on the right in (2.26) has two parts - the integral over the boundary at infinity $\partial_{\infty} S$, and over the nuts and bolts $\partial S$; these will be treated separately. 
First, for the integral at infinity, using the fact that $S$ approximates $\Sigma$ near infinity, by prior estimates, we have

$$
|\omega|=O\left(r^{-1}\right),|\kappa|=O(r), u^{2}=O\left(r^{2}\right), \text { and area } S(r)=O\left(r^{2}\right) .
$$

It follows that the boundary integrand at infinity in (2.26) is bounded. Further, (2.25) implies that for any unit tangent vector in $T S,(\kappa /|\kappa|)(e) \rightarrow 0$ at infinity. Hence the boundary integral at infinity vanishes.

For the boundary integral over the bolts and nuts, as noted in (2.8) and (2.9), the integral of $u^{-3}|\omega|$ over bolts and nuts in the orbit space $\Sigma$ is finite, i.e. the integral over the boundary of an $\varepsilon$-tubular neighborhood tends to a finite limit as $\varepsilon \rightarrow 0$. Since the volume of such a boundary approximation in the manifold $M$ is small compared with that in $\Sigma$, and since in addition $|\kappa|$ is bounded, ( $|\kappa| \rightarrow 0$ in fact), it is clear that the boundary integral over bolts and nuts also vanishes.

It follows then from $(2.26)$ that $\omega \equiv 0$ on $S$. Since $\omega$ is also invariant under the $S^{1}$ action, it follows that $\omega \equiv 0$ on $M$. This implies that $(M, g)$ is everywhere locally static.

We complete this section with the following topological result, needed for the work in the next section. []

Lemma 2.6 Let $M$ be a compact 4-manifold with boundary. Suppose $M$ has a topologically static $S^{1}$ action $\Phi_{0}: S^{1} \rightarrow \operatorname{Diff}(M)$ without exceptional orbits. If $\Phi_{1}: S^{1} \rightarrow \operatorname{Diff}(M)$ is any other $S^{1}$ action obtained by deformation from $\Phi_{0}$, i.e. there is a continuous curve $\Phi_{t}$ of $S^{1}$ actions joining $\Phi_{0}$ and $\Phi_{1}$, then $\Phi_{1}$ is also topologically static without exceptional orbits.

Proof: This follows from the topological classification theorem in [15]. Namely, smooth $S^{1}$ actions on $M$ are uniquely determined, (up to smooth conjugacy), by discrete data associated to the orbit space $\Sigma$. But such data do not vary under continuous deformation of the action.

More directly, the structure of the orbits near fixed points, together with elementary topological considerations, shows that both the existence of the set of fixed points, its topology, as well as the topological properties of the orbit space near this set are stable under continuous deformations of the metric. It is then easy to reduce the problem to one where $\Phi_{0}$ has no fixed points. The properties just mentioned imply then that none of the $\Phi_{t}$ 's has fixed points. The openness of the property "having a slice" is obvious. To show that this property is closed, consider a sequence of actions $\Phi_{t_{i}}$ with $t_{i}$ converging to $t_{*}$. For each $t_{i}$ the orbit space is a trivial principal bundle, hence has vanishing characteristic class. By continuity, the $t_{*}$-orbit space will therefore also be a principal bundle with vanishing characteristic class, consequently a trivial principal bundle, as desired.

\footnotetext{
${ }^{9}$ Useful discussions with A.Zeghib concerning this Lemma are acknowledged.
} 
More general results of this kind may well hold; however Lemma 2.6 suffices for our needs. Note that it is easy to construct stationary and not static $S^{1}$ actions on any 4-manifold which admits topologically static $S^{1}$ actions.

\section{$3 \quad$ Existence and Uniqueness}

In this section, we apply the results of Section 2 to construct large classes of non-trivial static AdS vacuum space-times. In addition, several uniqueness results are proved.

Throughout this section, it is assumed that $(\partial M, \gamma)$ has a free globally static isometric $S^{1}$ action, i.e. $(\partial M, \gamma)$ is strictly globally static. Hence $\partial M$ is topologically of the form

$$
\partial M=S^{1} \times V,
$$

where $V$ is a closed surface. We work first in the Riemannian setting, and pass later to Lorentzian metrics. The metric $\gamma$ on $\partial M$ is of the form

$$
\gamma=\alpha^{2} d \phi^{2}+g_{V}
$$

with $\alpha>0$ the length of the $S^{1}$ orbits. Modulo these restrictions, the metric $\gamma$ may a priori be arbitrary, i.e. $\alpha$ and $g_{V}$ are arbitrary. It is worthwhile to make a brief dimension count here. The metric $\gamma$ corresponds to 2 free functions on $V$, after dividing out by diffeomorphisms of $V$; namely $\alpha$ and a conformal factor for $g_{V}$. Thus, $[\gamma]$, after dividing by diffeomorphisms, corresponds to 1 free function on $V$.

Let $\mathcal{C}_{S}=\mathcal{C}_{S}(\partial M) \subset \mathcal{C}(\partial M)$ be the space of pointwise conformal classes of such static boundary metrics. One can choose representatives of the conformal class in several natural ways; by requiring that $g_{V}$ is of constant curvature, by requiring $\alpha=1$, or by requiring $\gamma$ is a Yamabe metric on $\partial M$. Of course generally these normalisation are all distinct. Observe that the space $\mathcal{C}_{S}$ is connected.

Let $M$ be any compact, connected oriented 4-manifold, with boundary equal to $\partial M$, and let $\mathcal{E}_{S}=\mathcal{E}_{S}(M)$ be the moduli space of AH Einstein metrics on $M$, with boundary metrics in $\mathcal{C}_{S}$, i.e.

$$
\Pi^{-1}\left(\mathcal{C}_{S}\right)=\mathcal{E}_{S}
$$

for $\Pi$ the boundary map as in (2.1).

By Proposition 2.1, any AH Einstein filling metric $(M, g) \in \mathcal{E}_{S}$ of $(\partial M, \gamma)$, (if it exists), has an effective isometric $S^{1}$ action. If this action is topologically static on $M$, then Theorem 2.2 implies that the $S^{1}$ action on $(M, g)$ is static. Finally, Lemma 2.6 shows that the condition that the $S^{1}$ action is topologically static without exceptional orbits is stable, i.e. preserved under deformations. (This holds actually regardless of any equations.) It is clear that the isometric $S^{1}$ action induced on any given $g \in \mathcal{E}_{S}$ varies continuously with any continuous variation of $g$ in $\mathcal{E}_{S}$. It follows that on any given component $\mathcal{E}_{S}^{c}$ of $\mathcal{E}_{S}$, either every metric $g \in \mathcal{E}_{S}^{c}$ is globally static without exceptional orbits, or no $g \in \mathcal{E}_{S}^{c}$ 
has this property. Similarly the topology of the set of fixed points of the action is constant on any connected component of $\mathcal{E}_{S}^{c}$.

In the following, we always work on a globally static component $g \in \mathcal{E}_{S}^{c}$ of $\mathcal{E}_{S}$, and for simplicity write $\mathcal{E}_{S}^{c}$ as $\mathcal{E}_{S}$, when there is no danger of confusion. The metric $g \in \mathcal{E}_{S}$ then has the form

$$
g=u^{2} d \phi^{2}+g_{\Sigma}
$$

of a warped product on $M=\Sigma \times{ }_{u} S^{1}$, modulo diffeomorphisms in $\mathcal{D}_{1}$, i.e. equal to the identity on $\partial M$. In particular, there are no exceptional orbits or nuts $\$$, only bolts, (if $u$ vanishes somewhere). The $S^{1}$ action is given by rotation about the $S^{1}$ factor and the 3-manifold $\Sigma$ has boundary at infinity given by $\partial_{\infty} \Sigma=V$. The boundary map (2.1) thus naturally restricts to the boundary map

$$
\Pi_{S}: \mathcal{E}_{S} \rightarrow \mathcal{C}_{S}
$$

on globally static components $\mathcal{E}_{S}^{c} \subset \mathcal{E}_{S}$. Both spaces $\mathcal{C}_{S}$ and $\mathcal{E}_{S}$ are smooth infinite dimensional Banach manifolds, (again assuming $\mathcal{E}_{S} \neq \emptyset$ ). In fact both are closed submanifolds of $\mathcal{C}$ and $\mathcal{E}$ respectively. The restricted boundary map $\Pi_{S}$ is again a smooth Fredholm map of Fredholm index 0.

Next, let $\mathcal{C}^{o}$ be the space of conformal classes of metrics on $\partial M$ containing non-flat representative of non-negative scalar curvature. Similarly, let $\mathcal{C}_{S}^{o}$ be the corresponding space of strictly globally static boundary metrics. While it is unknown if $\mathcal{C}^{o}$ is connected or not, the space $\mathcal{C}_{S}^{o}$ is connected, by the uniformization theorem for surfaces. As in (3.3), define

$$
\mathcal{E}^{o}=\Pi^{-1} \mathcal{C}^{o}, \mathcal{E}_{S}^{o}=\Pi^{-1}\left(\mathcal{C}_{S}^{o}\right)
$$

By $\llbracket$, Thm.B], the boundary maps $\Pi$ and $\Pi_{S}$, restricted to $\mathcal{E}^{o}$ and $\mathcal{E}_{S}^{o}$ respectively, are proper maps, provided the map on homology induced by the inclusion, $H_{2}(\partial M, \mathbb{R}) \rightarrow H_{2}(M, \mathbb{R})$ is surjective. (This will be the case in all situations discussed below). In particular, there are only finitely many components of $\mathcal{E}^{o}$ and $\mathcal{E}_{S}^{o}$. As above, in the following, we will always work in a fixed globally static component of $\mathcal{E}_{S}^{o}$.

Moreover, the maps $\Pi$ and $\Pi_{S}$ have a well-defined degree on $\mathcal{E}^{o}$ and $\mathcal{E}_{S}^{o}$, with

$$
\operatorname{deg} \Pi=\operatorname{deg} \Pi_{S} \in \mathbb{Z} .
$$

The degree is computed by counting, with suitable signs, the number of points in the inverse image of a regular value of $\Pi$, resp. $\Pi_{S}$. (Of course the degree may change on different components of $\mathcal{E}^{o}$ ).

All of this discussion now carries over to the Lorentzian setting, by changing $d \phi^{2}$ to $-d t^{2}$ and unwrapping the $S^{1}$ to $\mathbb{R}$. The boundary metric then has the Lorentz form

$$
\gamma=\gamma^{L}=-\alpha^{2} d t^{2}+g_{V}
$$

Further, it is clear that each AH (Riemannian) Einstein metric (3.4) corresponds uniquely to a Lorentz metric of the form

$$
\mathfrak{g}=-u^{2} d t^{2}+g_{\Sigma}
$$


and vice versa. The metric $\mathfrak{g}$ is a solution to the vacuum Einstein equations with $\Lambda=-6$. The bolts $B=\partial \Sigma=\{u=0\}$ correspond to a horizon in the resulting space-time in the usual way.

We now separate the discussion into distinct cases, according to the genus of $V$.

\section{$3.1 \quad V=S^{2}$.}

Then $\partial M=\mathbb{R} \times S^{2}$, or $S^{1} \times S^{2}$ in the Riemannian setting. Working first in the Riemannian setting, suppose that

$$
M=S^{1} \times \mathbb{R}^{3} .
$$

The manifold $M$ carries a strictly globally static AH Einstein metric, namely the hyperbolic metric $g_{0}$ on $H^{4}(-1) / \mathbb{Z}$, where the action of $\mathbb{Z}$ is generated by a hyperbolic translation along a geodesic. The $S^{1}$ action on $M$ and $\partial M$ are given by rotation in the $S^{1}$ factor in (3.8). Let $\mathcal{E}, \mathcal{E}_{S}$ and $\mathcal{E}_{S}^{o}$ be the corresponding components containing $g_{0}$. Hence, by the discussion above, $\mathcal{E}_{S}$ and $\mathcal{E}_{S}^{o}$ consist of globally static AH Einstein metrics on $M$.

Existence: By [4, $\S 7$, for $M$ as in (3.8),

$$
\operatorname{deg} \Pi=\operatorname{deg} \Pi_{S}=1 .
$$

Since $\operatorname{deg} \Pi \neq 0$ implies $\Pi$ is surjective, it follows that any $C^{m, \beta}$ boundary metric of non-negative scalar curvature is the boundary metric of an $\mathrm{AH}$ Einstein metric on $M, m \geq 4$. More to the point here, $\operatorname{deg} \Pi_{S} \neq 0$ implies that $\Pi_{S}$ is surjective onto $\mathcal{C}_{S}^{o}$, and hence any class in $\mathcal{C}_{S}^{o}$ is the conformal infinity of a globally static AH Einstein metric on $M$. Observe that

$$
u>0 \text { on } M
$$

so that $(M, g)$ is in fact strictly globally static. This follows by a simple topological argument. Namely suppose $u(p)=0$ somewhere in $M$, and for $\exp (i \alpha) \in S^{1}$, $q \in S^{1} \times \mathbb{R}^{3}$ let $\phi(\alpha, q)$ denote the action of $S^{1}$ on $S^{1} \times \mathbb{R}^{3}$. By construction the orbits $\alpha \rightarrow \phi(\alpha, q)$ are homotopically non-trivial circles for all $q$ near the conformal boundary. Let $q$ be such a point and for $s \in[0,1]$ let $\gamma(s)$ be any continuous path from $p$ to $q$. Then $\phi(\alpha, \gamma(s))$ provides a continuous deformation of $\{\phi(\alpha, \gamma(1)=q)\}_{\alpha \in[0,2 \pi]}$ to $\{p\}=\{\phi(\alpha, \gamma(0)=p)\}_{\alpha \in[0,2 \pi]}$, which is not possible.

Passing to the Lorentzian setting, the following corollary - which implies Theorem 1.1 - is now immediate.

Corollary 3.1 Any $C^{m, \beta}, m \geq 4$, globally static boundary metric

$$
\gamma=-\alpha^{2} d t^{2}+g_{S^{2}}
$$

with $\alpha>0$ and of non-negative scalar curvature on $\mathbb{R} \times S^{2}$, is the conformal infinity of a complete, strictly globally static vacuum AdS metric on $\mathbb{R} \times \mathbb{R}^{3}=$ $\mathbb{R}^{4}$. 
Uniqueness: It is not known in general whether there is a unique static Riemannian, (or Lorentzian), Einstein metric on $\left(S^{1} \times \mathbb{R}^{3}, g\right)$ filling in a given boundary metric $\gamma \in \mathcal{C}^{o}$. The boundary map $\Pi_{S}$ is of degree 1 . The Sard-Smale theorem [32] implies that the regular values of $\Pi_{S}$ are generic, and in particular dense, in $\mathcal{C}_{S}^{o}$. Hence a generic boundary metric in $\mathcal{C}_{S}$ has an odd number of Einstein filling metrics in the component $\mathcal{E}_{S}^{o}$. There are at present however no good arguments in favor of uniqueness in general.

On the other hand, in the presence of more symmetry, one can obtain uniqueness; a similar result has been already established in [12] (extending a previous argument of [9]) by completely different methods:

Proposition 3.2 The standard anti-de Sitter metric $g_{o}$ on $\mathbb{R} \times \mathbb{R}^{3}$ is the unique globally static AdS vacuum metric on $\mathbb{R} \times \mathbb{R}^{3}$ which admits a $C^{2}$ (or $L^{2, p}, p>4$ ) conformal completion, with a conformally compactifiable smooth acausal hypersurface orthogonal to the static Killing field, and which has boundary metric $\gamma_{0}=-d t^{2}+g_{+1}$, where $g_{+1}$ is the round metric on $S^{2}$.

Proof: By the correspondence Lorentzian - Riemannian discussed above, it suffices to prove the Riemannian analogue of this. This has already been done in [4, §7], using the fact that the large isometry group $S^{1} \times S U(2)$ of the Riemannian boundary metric extends to the same isometry group of any $\mathrm{AH}$ Einstein metric, together with the classification of Einstein metrics with such a large isometry group on the given manifold.

Next, for $V=S^{2}$ as before, assume that

$$
M=\mathbb{R}^{2} \times S^{2}
$$

in the Riemannian setting. The manifold $M$ carries a natural globally static AH Einstein metric, namely the Riemannian (or Euclidean) Schwarzschild metric

$$
g_{\mathrm{Schw}}=u^{2} d \phi^{2}+u^{-2} d r^{2}+r^{2} g_{S^{2}(1)},
$$

where $u^{2}=1+r^{2}-\frac{2 m}{r}, m>0$. The period of $\phi$ depends on $m$, see the discussion below following (3.13). The boundary metric $\gamma_{0}$ of $g_{\text {Schw }}$ is clearly in $\mathcal{C}_{S}^{o}$ while $g_{\text {Schw }} \in \mathcal{E}_{S}^{o}$, where the $S^{1}$ action is given by rotation in the $\phi$-circles. Hence, as above, the full component $\mathcal{E}_{S}^{o}$ containing $g_{\mathrm{Schw}}$ consists of globally static AH Einstein metrics on $M$.

For simple topological reasons as before, any metric in $\mathcal{E}_{S}^{o}$ has a unique bolt $B, B=S^{2}$.

Existence: By $\llbracket$ 四, 7$]$,

$$
\operatorname{deg} \Pi=0
$$

and $\Pi$ is not onto $\mathcal{C}^{o}$. Similarly, $\Pi_{S}$ is not onto $\mathcal{C}_{S}^{o}$. In addition, uniqueness also fails, in that for any regular value of $\gamma \in \mathcal{C}^{o}$ there are an even number, (possibly 0 ), of Einstein filling metrics. 
Nevertheless, the moduli spaces $\mathcal{E}$ and $\mathcal{E}_{S}$ are non-empty, since the Schwarzschild metrics (3.11) are in $\mathcal{E}_{S}$. Further, for a generic value of the mass $m$, the Schwarzschild metrics are regular points of $\Pi$, cf. [20] and hence $\Pi(\mathcal{E})$ and $\Pi\left(\mathcal{E}_{S}\right)$ are varieties of codimension 0 , (containing open sets), in $\mathcal{C}$ and $\mathcal{C}_{S}$ respectively.

To any metric $g \in \mathcal{E}_{S}$ is associated a globally static Lorentzian-Einstein metric on the manifold

$$
\mathscr{M}=\mathbb{R} \times\left(\mathbb{R}^{+} \times S^{2}\right),
$$

with metric of the form (3.7). The usual extensions of the space-time lead to smooth horizons, topologically $\mathbb{R} \times S^{2}$, within $\mathscr{M}$.

This gives the existence of a large, infinite dimensional, family of smooth, conformally compact vacuum AdS black hole metrics on $\mathscr{M}$ as in (3.12). This is in marked contrast to the black hole uniqueness theorem in case $\Lambda=0$. Of course the main example is the AdS Schwarzschild metric, (or spherical Kottler metric),

$$
g_{\mathrm{Schw}}=-u^{2} d t^{2}+u^{-2} d r^{2}+r^{2} g_{S^{2}(1)} .
$$

We point out however an interesting difference in the behavior of the boundary maps $\Pi$ in the Riemannian and Lorentzian settings. Thus, note that as $m$ varies, the conformal class of the boundary metric of the Riemannian $g_{\text {Schw }}$ in (3.11) varies; the boundary metric is of the form

$$
d \phi^{2}+g_{S^{2}(1)}
$$

for $\phi \in[0, \beta]$, where $\beta=4 \pi r_{+} /\left(1+3 r_{+}^{2}\right)$, and $r_{+}$is the smallest root of $u^{2}$. This condition on the period of $\phi$ is due to the requirement of smoothness of the metric at the bolt.

In the Lorentzian case where $\phi$ is unwrapped to $t$, there is no such restriction and the full 1-parameter family of Schwarzschild metrics $g_{\mathrm{Schw}}(m)$ has the same boundary metric, i.e.

$$
-d t^{2}+g_{S^{2}(1)}
$$

The same feature essentially holds for general globally static metrics on $M$ in (3.10). Namely, choose a Riemannian static boundary metric in $\operatorname{Im} \Pi_{S}$,

$$
\gamma=\gamma^{R}=\alpha^{2} d \phi^{2}+g_{S^{2}}
$$

Suppose a neighborhood of $[\gamma]$ in $\mathcal{C}_{S}$ is also in $\operatorname{Im} \Pi_{S}$, cf. the remarks above on the structure of the variety $\Pi_{S}\left(\mathcal{E}_{S}\right) \subset \mathcal{C}_{S}$. In this case, one can vary $\alpha$ by $\alpha \rightarrow(1+s) \alpha$, for $s \in(-\varepsilon, \varepsilon)$, while remaining in $\operatorname{Im} \Pi_{S}$. This gives a curve of distinct boundary metrics $\gamma_{s}^{R}$, or more precisely, a non-trivial curve in the space of conformal classes $\mathcal{C}_{S}$. This curve gives rise to a curve of isometrically distinct filling Einstein metrics $g_{s}^{R}$ in the Riemannian case. Hence, one also has a curve $g_{s}^{L}$ of isometrically distinct Lorentzian-Einstein metrics.

However, the corresponding curve of Lorentzian boundary metrics $\gamma_{s}^{L}$ are all isometric, in that there is a curve of diffeomorphisms $\psi_{s}$ of $\partial M,\left(\psi_{s} \neq i d\right)$, such that $\left(\psi_{s}^{L}\right)^{*} \gamma_{0}^{L}=\gamma_{s}^{L}$. Namely,

$$
\gamma_{s}=-\alpha^{2}(1+s)^{2} d t^{2}+g_{S^{2}}=\psi_{s}^{*}\left(\gamma_{0}\right)
$$


where $\psi_{s}(t, q)=((1+s) t, q), q \in S^{2}$.

Thus, if one identifies such boundary metrics differing only by a dilation of the $t$ factor, one then has local 1-parameter families of distinct AdS globally static vacuum Lorentz metrics with the same boundary metric. Presumably, the 1-parameter comes from an appropriate notion of mass.

Regarding uniqueness, we have the following analogue of the black hole uniqueness theorem when $\Lambda=0$, or analogue of the rigidity of anti-de Sitter space in Proposition 3.2.

Proposition 3.3 The AdS Schwarzschild metrics $g_{\mathrm{Schw}}(m)$ are the unique globally static vacuum metrics on $\mathbb{R} \times\left(\mathbb{R}^{+} \times S^{2}\right)$, smooth up to the horizon, which admit a $C^{2}$ (or $L^{2, p}, p>4$ ) conformal completion, with a conformally compactifiable smooth acausal hypersurface orthogonal to the static Killing field, with boundary metric $\gamma_{0}=-d t^{2}+g_{+1}$, where $g_{+1}$ is the round metric on $S^{2}$.

Proof: The proof is the same as that of Proposition 3.2. Thus, it suffices to prove this in the Riemannian setting, and this has already been done in [ [ 1 , $\S 7$, using the fact that any $\mathrm{AH}$ Einstein filling metric has an isometric $S^{1} \times S U(2)$ action.

Propositions 3.2 and 3.3 have the following important corollary:

TheOREM 3.4 The standard AdS metric and the domain of outer communication of the AdS Schwarzschild metrics $g_{\text {Schw }}(m), m>0$, are the unique static globally hyperbolic (in the sense of manifolds with boundary) vacuum metrics which admit $C^{2}$ (or $L^{2, p}, p>4$ ) conformal completions, with conformally compactifiable acausal spacelike surfaces (with perhaps an interior boundary on which u vanishes), without degenerate event horizons, and with boundary metric (at infinity) $\gamma_{0}=-d t^{2}+g_{+1}$, where $g_{+1}$ is the round metric on $S^{2}$.

Proof: Global hyperbolicity implies that $\mathfrak{g}$ has the global representation (3.7), so that the Riemannian counterpart of $(\mathscr{M}, \mathfrak{g})$ satisfies all the hypotheses of Section 2. (The non-degeneracy of the event horizons ensures compactness of the associated Riemannian manifold, compare [11, Section 3].) The large isometry group forces the topology of the filling space-time to be that considered in Propositions 3.2 or 3.3 , and the result immediately follows.

As usual the above claim concerns only the domain of outer communication. No natural reasonable conditions are known which would guarantee some form of uniqueness beyond the event horizon. We note that there are at least two ways of adding a boundary $\{u=0\}$ to the set $\{u>0\}$ on the Cauchy surface: either by gluing in a sphere (which becomes a boundary for the new manifold), or by gluing in a $\mathbb{R P}^{2}$ (which becomes an interior surface for the new manifold).

\section{$3.2 \quad V=T^{2}$.}

Then $\partial \mathscr{M}=\mathbb{R} \times T^{2}$, or $\partial M=S^{1} \times T^{2}=T^{3}$ in the Riemannian setting. First consider the Riemannian setting. As filling manifold, we choose

$$
M=D^{2} \times T^{2},
$$


where $D^{2}$ is a disc filling some $S^{1} \subset T^{3}=\partial M$. This manifold carries the "generalised Riemannian AdS $T^{2}$ black hole" metrics

$$
g_{T^{2}}(m)=\left[u^{-2} d r^{2}+\left(u^{2} d s^{2}+r^{2} \gamma_{0}\right)\right] / \Gamma
$$

where $u^{2}=\left(r^{2}-\frac{2 m}{r}\right), m>0, \gamma_{0}$ is the flat metric on $\mathbb{R}^{2}$, and $\Gamma$ is any flat lattice in $\mathbb{R}^{3}=\mathbb{R} \times \mathbb{R}^{2}$. Here $\Gamma$ is arbitrary, subject to the constraint that the $S^{1} \subset \mathbb{R}^{3} / \Gamma$ bounding the disc $D^{2}$ in (3.14) has $\gamma_{0}$-length depending on $m$, to give a smooth metric where $u=0$; this is the same issue as the constraint on $\beta$ following (3.13). Alternately, prescribing the $\gamma_{0}$-length determines $m$. The action of $\Gamma$ on $\mathbb{R}^{3}$ has many distinct extensions to an action of $\Gamma$ on the universal cover $\widetilde{M}=D^{2} \times \mathbb{R}^{2}$, cf. [3, $\S 4.3$ and 4.4] for further details.

A simple computation shows that the metrics (3.15) all have non-positive sectional curvature and hence they are regular points of $\Pi$, cf. [28,7]. In fact, the metrics $g_{T^{2}}$ are all locally isometric. Thus, as before, $\Pi(\mathcal{E})$ is a variety of codimension 0 in $\mathcal{C}$.

However, in general, metrics of the form (3.15) are not globally static w.r.t. any $S^{1}$ action - they are only locally static. Moreover, they are locally static w.r.t. many $S^{1}$ actions. Because the proof uses global arguments, we do not know if Theorem 2.2 holds for locally static $S^{1}$ actions on $\partial M$, (although we expect this to be the case).

In this situation, $\mathcal{C}^{o}=\emptyset$ - there are no non-flat metrics of non-negative scalar curvature on $\partial M=T^{3}$, and the degree theory breaks down. In fact the boundary map $\Pi$ is not proper on flat boundary metrics $\gamma_{0}$ on $T^{3}=S^{1} \times T^{2}$; there are infinitely many distinct AH Einstein metrics $g_{i}=g_{i}\left(\gamma_{0}\right)$, all of the form (3.15) with a given conformal infinity $\left(S^{1} \times T^{2}, \gamma_{0}\right)$, for any fixed flat metric $\gamma_{0}$. The infinite sequence comes from the infinite number of ways $S^{1} \subset \partial M$ may bound a disc $D^{2}$ in (3.14), or equivalently the infinite number of extensions of a given $\Gamma$ action to an action on $\widetilde{M}$. We point out that these metrics $g_{i}$ lie in distinct components $\mathcal{E}_{S}^{c}$ of the full moduli space $\mathcal{E}_{S}$ in (3.3). Thus, $\mathcal{E}_{S}$ in fact has infinitely many distinct components $\mathcal{E}_{S}^{c}$, each consisting of static $\mathrm{AH}$ Einstein metrics on $M$, cf. again [3, §4.4], for further details.

Because the degree theory breaks down, there is currently no general existence theorem for AH Einstein metrics on $M$ with prescribed conformal infinity, as in Corollary 3.1. Nevertheless, as mentioned above, each component $\mathcal{E}_{S}^{c}$ of $\mathcal{E}_{S}$ containing a $T^{2}$ black hole metric is a large, infinite dimensional space, with $\Pi\left(\mathcal{E}_{S}^{c}\right)$ containing open sets of $\mathcal{C}_{S}$.

We turn to the Lorentzian situation below, but first prove the following uniqueness theorem for Riemannian AH Einstein metrics with a flat boundary metric on $T^{3}$.

Proposition 3.5 Let $\gamma_{0}$ be a flat metric on $\partial M=T^{3}=S^{1} \times S^{1} \times S^{1}$. Then any AH Einstein metric with conformal boundary class $\left[\gamma_{0}\right]$ is of the form (3.15). Further, for every $\gamma_{0}$ there exists precisely a countable infinity of distinct filling AH Einstein metrics (3.15), each member of this family being uniquely determined by specifying which $S^{1} \subset T^{3}$, (not necessarily a product factor), bounds the disc $D^{2}$ in (3.14). 
Proof: Any AH Einstein metric $g$ on a manifold $N$ with conformal infinity a flat metric on $T^{3}$ has an effective isometric $T^{3}$ action, by Proposition 2.1. In particular, the principal orbits of this action are all flat tori, and so the manifold $N$ must be of the form $M$ in (3.14). On the universal cover the metric takes thus the form

$$
g_{r r}(r) d r^{2}+g_{i j}(r)\left(d x^{i}+U^{i}(r) d r\right)\left(d x^{j}+U^{j}(r) d r\right),
$$

with $r$ in an interval $I \subset \mathbb{R}$. We wish to show that without loss of generality the metric can be taken to be diagonal ${ }^{10}$. Choose $r_{0} \in I$, a change of coordinates $x^{i} \rightarrow y^{i}=x^{i}+\int_{r_{0}}^{r} U^{i}(s) d s$ sets the $U^{i}$ 's to zero. A reparameterization of $I$ leads to $g_{r r}=1$. By a linear change of coordinates we can achieve $g_{i j}\left(r_{0}\right)=\delta_{i}^{j}$. A further ( $r$-independent) rotation of the coordinates leads to $\partial_{r} g_{i j}\left(r_{0}\right)=\operatorname{diag}\left(a_{0}^{2}, b_{0}^{2}, c_{0}^{2}\right)$. Now, the Einstein equation for $g$ implies a second order system of ODE's for $g_{i j}$ (for the reader familiar with general relativity, these are the usual ADM evolution equations with zero shift and with the square of the lapse function replaced by -1 ), giving a unique solution for every set of strictly positive numbers $a_{0}, b_{0}, c_{0}$; this set will be referred to as "set of equations GE". (Here we do not normalize the scalar curvature, demanding only strict negativity, so that there is no "scalar constraint equation".) On the other hand, the equations which are obtained by assuming that the metric is diagonal (see e.g. 29]) show that there also exists a solution with $g_{i j}(r)$ - diagonal for all $r$. Uniqueness of solutions of the "set of equations GE" implies that in the above coordinate system $g_{i j}(r)$ is indeed diagonal. It follows that there exists a coordinate system in which the metric $g$ is of the form

$$
d r^{2}+a^{2}\left(\theta^{1}\right)^{2}+b^{2}\left(\theta^{2}\right)^{2}+c^{2}\left(\theta^{3}\right)^{2},
$$

where $a, b, c$ are functions of $r$ only, while the $\theta^{i}$, s form a constant coefficients basis of the space of one-forms anihilating $\partial_{r}$. It is now a straightforward computation to see that the only such metrics which give a smooth Einstein conformally compactifiable metric on $M$ are of the form 3.15) (compare [29, [23). This justifies uniqueness.

As in the discussion prior to the proposition, there is a countable infinity of possible choices of which simple closed geodesic $\Gamma \subset T^{3}$ will bound the disc $D^{2}$ occurring in (3.14), and each such choice will lead to a different filling metric, uniquely determined by $\Gamma$, as follows:四 let $\gamma_{0}$ be an arbitrary flat metric on $T^{3}$, and let $\Gamma \approx S^{1} \subset T^{3}$ be the simple closed geodesic which will be filled by a $D^{2}$. Let $\varphi \in[0,2 \pi]$ be a $2 \pi$ periodic affine parameter on $\Gamma$, and let $\left(\varphi_{1}, \varphi_{2}, \varphi_{3}\right) \in[0,2 \pi]^{3}$ be a coordinate system on $T^{3}$ such that $\left.\varphi_{1}\right|_{\Gamma} \equiv \varphi,\left.\varphi_{2}\right|_{\Gamma}=$ $\left.\varphi_{3}\right|_{\Gamma}=0$, and such that the one-forms $d \varphi_{i}$ are $\gamma_{0}$-covariantly constant. Let $\theta^{i}$

\footnotetext{
${ }^{10}$ The argument given here is a straightforward rephrasing of the usual argument that Bianchi I metrics are diagonal.

${ }^{11}$ An alternative way of understanding the non-uniqueness is as follows: any flat metric $\gamma$ on $T^{3}$ can be represented by a constant coefficient matrix $\gamma=\gamma_{i j} d \varphi^{i} d \varphi^{j}$. The representation is unique up to a linear relabeling of the $\varphi^{i}$ 's, $\varphi^{i} \rightarrow L^{i}{ }_{j} \varphi^{j}$, with $L^{i}{ }_{j} \in S L(3, \mathbb{Z})$. If we decree that the $D^{2}$ factor in (3.14) fills the first $S^{1}$ factor of $T^{3}$, then each choice of $L^{i}{ }_{j}$ will lead to a different filling metric.
} 
be a $\gamma_{0}$-orthonormal co-frame on $T^{3}$ with $\theta^{2}\left(\partial_{\varphi_{1}}\right)=\theta^{3}\left(\partial_{\varphi_{1}}\right)=0$. Rescaling $\gamma_{0}$ by a constant factor if necessary we can assume that the $\gamma_{0}$-length of $\Gamma$ equals $2 \pi$, thus $\theta^{1}\left(\partial_{1}\right)=1$. Parameterising $D^{2}$ by polar coordinates $\left(\rho, \varphi_{1}\right) \in$ $[0,1) \times[0,2 \pi]$ we extend the $\theta^{i}$ 's to $D^{2} \times T^{2}$ so that $\mathscr{L}_{\partial_{\rho}} \theta^{i}=\theta^{i}\left(\partial_{\rho}\right)=0$. Let $r:[0,1) \rightarrow[1 / 2, \infty)$ be any diffeomorphism of $[0,1)$ with $[1 / 2, \infty)$, then the desired smooth filling metric is

$$
g=u^{-2} d r^{2}+u^{2}\left(\theta^{1}\right)^{2}+r^{2}\left(\left(\theta^{2}\right)^{2}+\left(\theta^{3}\right)^{2}\right),
$$

with

$$
u^{2}(r)=r^{2}-2 m / r, \quad m=2^{-4} .
$$

Now we turn to the Lorentzian setting, still assuming that $\partial M=S^{1} \times T^{2}$, so that $\partial \mathscr{M}=\mathbb{R} \times T^{2}$. Suppose first that the associated Lorentzian space-time $\mathscr{M}$ takes the form

$$
\mathscr{M}=\mathbb{R} \times\left(D^{2} \times S^{1}\right),
$$

with $M=S^{1} \times\left(D^{2} \times S^{1}\right)$. Here the static action on $\partial M$ comes from a free globally static $S^{1}$ action on the $T^{2}$ factor in (3.14). Since the $S^{1}$ orbits do not bound a disc in $M$, it follows that the $S^{1}$ action is strictly globally static. Hence, the corresponding Lorentz metric on $\mathscr{M}$ as in (3.16) is a strictly globally static vacuum AdS metric and so has the form

$$
g=-u^{2} d t^{2}+g_{\Sigma}
$$

where $\Sigma=D^{2} \times S^{1}$ and $u>0$ on $\Sigma$.

The same remarks on the existence of a large space of such vacuum AdS solutions as in the Riemannian case apply here also.

The main examples in this situation are the Horowitz-Myers AdS soliton metrics, [22], in dimension 4:

$$
g_{T^{2}}(m)=-r^{2} d t^{2}+u^{-2} d r^{2}+\left(u^{2} d s_{1}^{2}+r^{2} d s_{2}^{2}\right) / \Gamma_{0},
$$

where $u^{2}=\left(r^{2}-\frac{2 m}{r}\right)$, and $\Gamma_{0}$ is any lattice acting on $\mathbb{R}^{2}=\mathbb{R}^{2}\left(s_{1}, s_{2}\right)$.

The Riemannian-Lorentzian correspondence and Proposition 3.5 then imply immediately the following uniqueness result for the AdS soliton metrics. This result generalises a recent result of Galloway, Surya and Woolgar 17, 16], in dimension four. We note that the $S L(3, \mathbb{Z})$ discrete degrees of freedom of Proposition 3.5 are reduced now to $S L(2, \mathbb{Z})$ discrete degrees of freedom:

Proposition 3.6 All conformally compactifiable static vacuum AdS metrics on $\mathbb{R} \times D^{2} \times S^{1}$ with acausal conformally compactifiable hypersurfaces orthogonal to the static Killing vector and with (conformal) boundary metric the flat product $-d t^{2}+\gamma_{0}$ on $\mathbb{R} \times T^{2}$ are of the form (3.18). Further, for any such $\gamma_{0}$ there is precisely a countable family of filling Einstein metrics (3.18), each member of this family being determined by specification of the $S^{1} \subset T^{2}$ (not necessarily a factor of the product $T^{2}=S^{1} \times S^{1}$ ) bounding the disc in (3.16). 
It seems of interest to make some comments about cylindrically symmetric, or axially symmetric, strictly globally static metrics. Let $\mathfrak{g}$ by any of the vacuum Lorentzian metrics on $\mathbb{R} \times D^{2} \times S^{1}$ mentioned after (3.17). Unwinding the last $S^{1}$-factor and calling $z$ the associated coordinate along $\mathbb{R}$ one obtains a $z$ periodic solution on $\mathbb{R} \times D^{2} \times \mathbb{R}=\mathbb{R} \times \mathbb{R}^{3}$. If the boundary metric is invariant under rotations of the $S^{1}=\partial D^{2}$ factor, then so is the filling metric, and we have thus obtained a $z$-periodic axi-symmetric solution. If, instead, the boundary metric is invariant under rotations along the last $S^{1}$-factor, the resulting filling metric will be invariant under $z$-translations. It is a corollary of Proposition 3.6 that all appropriately regular cylindrically symmetric strictly globally static solutions in the class considered here belong to the Horowitz-Myers family; the latter correspond to the cylindrically symmetric strings of Linet [29] with the constant $K$ in [29] equal to one.

Next, we turn to the case where there is a horizon. In this situation, the $S^{1}$ action on $\partial M$ is taken to be rotation in the $S^{1}$ factor bounding $D^{2}$. Hence, the Lorentzian manifold is of the form

$$
\mathscr{M}=\mathbb{R} \times\left(\mathbb{R}^{+} \times T^{2}\right) .
$$

The metric is of the form (3.17), but with a non-empty $\mathbb{R} \times T^{2}$ horizon $\{u=0\}$. Again the same remarks on the existence of a large infinite dimensional family of such $T^{2}$ AdS black hole metrics apply as before.

Similarly, the Riemannian-Lorentzian correspondence and Proposition 3.5 give immediately the following uniqueness theorem. Note that the discrete degrees of freedom of Proposition 3.5 have disappeared, since the $S^{1}$ bounding a disc has been chosen to correspond to the $\mathbb{R}$ factor:

Proposition 3.7 The AdS T $T^{2}$ black hole metrics

$$
g_{T^{2}}(m)=-u^{2} d t^{2}+u^{-2} d r^{2}+\left(r^{2} \gamma_{0}\right) / \Gamma_{0},
$$

where $u^{2}=\left(r^{2}-\frac{2 m}{r}\right), \gamma_{0}$ is the flat metric on $\mathbb{R}^{2}$, and $\Gamma_{0}$ is any lattice acting on $\mathbb{R}^{2}=\mathbb{R} \times \mathbb{R}$, are the unique globally static vacuum conformally compactifiable AdS metrics with a smooth acausal conformally compactifiable hypersurface orthogonal to the static Killing field (with a boundary on which $u=0$ ) and with boundary metric the flat metric $-d t^{2}+\mathbb{R}^{2} / \Gamma_{0}$.

As in the $S^{2}$ black hole case, as $m$ varies, the boundary metrics are fixed, for a given lattice.

Similarly to Theorem 3.4, we obtain:

TheOrem 3.8 The Horowitz-Myers metrics and the domains of outer communications of the toroidal Kottler metrics are the only static globally hyperbolic (in the sense of space-times with boundary) vacuum metrics which admit $C^{2}$ (or 
$\left.L^{2, p}, p>4\right)$ conformal completions, with conformally compactifiable Cauchy surfaces (with perhaps an interior boundary on which $u$ vanishes), without degenerate event horizons, and with boundary metric (at infinity) $\gamma_{0}=-d t^{2}+g_{0}$, where $g_{0}$ is a flat metric on $T^{2}$. In the strictly static case there is precisely one distinct filling Horowitz-Myers metric for each choice of an $S^{1} \subset T^{2}$ (not necessarily a product factor) which bounds a disc $D^{2}$ in the filling space-time.

A possible interpretation of Theorem 3.8 is that metrics as above are classified (up to discrete degrees of freedom) by their global (Hamiltonian) mass $M$ : negative $M$ leads to the Horowitz-Myers strictly static solutions, positive $M$ leads to the Kottler black hole solutions, solutions with zero $M$ do not exist. Note that $M$ is proportional to the parameter $m$ appearing in (3.20) in the black hole case, and proportional to minus the parameter $m$ appearing in (3.18) in the strictly static case. In the Horowitz-Myers case this point of view is somewhat misleading, as the mass itself is actually determined by $\left[\gamma_{0}\right]$ and the choice of the filling disc: thus, to every boundary class $\left[\gamma_{0}\right]$ one can uniquely associate a discrete sequence of masses.

\section{3 $V=\Sigma_{g}, g \geq 2$.}

Then $\partial M=\mathbb{R} \times \Sigma_{g}, S^{1} \times \Sigma_{g}$ in the Riemannian setting. Again, there exist no metrics of non-negative scalar curvature on $\partial M$, and so there is no degree theory.

There are no known strictly globally static AH Einstein metrics on $\mathbb{R} \times H_{g}$, where $H_{g}$ is a 3 -manifold with $\partial H_{g}=\Sigma_{g}$; (for instance $H_{g}$ a handlebody). It has been conjectured that in fact no such strictly globally static solutions exist.

On the other hand, suppose

$$
M=D^{2} \times \Sigma_{g}
$$

On such an $M$, one has the Riemannian genus $g$ AdS black hole metrics

$$
g=u^{2} d \phi^{2}+u^{-2} d r^{2}+r^{2} g_{-1},
$$

where $u^{2}=-1+r^{2}-\frac{2 m}{r}$, and $g_{-1}$ is a hyperbolic metric on $\Sigma_{g}$. (As before, the period of $\phi$ depends on $m$ ). These metrics are globally static, and so the full component $\mathcal{E}_{S}$ containing such metrics consists of globally static AH Einstein metrics on $M$. Each of these metrics has negative sectional curvature and so is a regular point of $\Pi$, as in the $T^{2}$ case. As before then, $\operatorname{Im} \Pi_{S}$ is a variety of codimension 0 in $\mathcal{C}_{S}$. Thus, again one has the existence of large, infinite dimensional space of such AH Einstein metrics, and a correspondingly large space of Lorentzian globally static AdS vacuum genus $g$ black hole metrics. These metrics are of the form (3.17) with $\Sigma=\Sigma_{g}$ and with horizon $\{u=0\}=$ $\mathbb{R} \times \Sigma_{g}$.

\footnotetext{
${ }^{12}$ The construction of the proof of Proposition 3.5 gives $m=2^{-4}$ for all metrics, in the normalisation of the boundary metric in which the bounding $S^{1}$ has prescribed length. However, when defining mass with $V=T^{2}$ one needs to normalise the $\gamma_{0}$-area of $T^{2}$; this will lead to distinct masses of the filling metrics once some such normalisation has been chosen.
} 
A uniqueness theorem as in Propositions 3.2, 3.3, etc. for such metrics with constant curvature boundary metric on $\Sigma_{g}$ remains currently an open problem however. This is because the symmetries of $\left(\Sigma_{g}, g_{-1}\right)$ are only local symmetries, and the analogue of Proposition 2.1 in this context, (i.e. for local symmetries), remains open.

\section{Properties of the Lorentzian solutions}

Consider any strictly globally static Lorentzian metric as constructed in the previous section, thus we have $\mathscr{M}=\mathbb{R} \times \Sigma$ with the vacuum metric

$$
\mathfrak{g}=-u^{2} d t^{2}+g_{\Sigma}
$$

as in (3.7). We wish to justify the properties of $(\mathscr{M}, \mathfrak{g})$ listed in the Introduction. Now, point 1 follows by construction. Point 6 follows from what has been said in the proof of Theorem 2.2. Point 2 is a straightforward corollary of point 6 . The geodesic completeness of the static metrics so obtained is a special case of the following result:

THEOREM 4.1 Let $\bar{\Sigma}=\partial \Sigma \cup \Sigma$ be a compact manifold with boundary. Consider a space-time $(\mathscr{M}=\mathbb{R} \times \Sigma, \mathfrak{g})$ such that

$$
\mathfrak{g}=-V^{2} d t^{2}+g_{\Sigma}, \quad \mathscr{L}_{X} V=\mathscr{L}_{X} g_{\Sigma}=g_{\Sigma}(X, \cdot)=0
$$

$X=\partial_{t}$, with $V$ strictly bounded away from zero. Suppose that $V^{-2} g_{\Sigma}$ can be extended by continuity to a differentiable Riemannian metric $\bar{g}_{\Sigma}$ on $\bar{\Sigma}$, such that $\partial \Sigma$ is totally geodesic in $\left(\Sigma, \bar{g}_{\Sigma}\right)$. We also assume that $V^{-1}$ can be extended by continuity to a differentiable function $\Omega$ on $\bar{\Sigma}$, with $\Omega$ vanishing precisely on $\partial \Sigma$ and $d \Omega$ nowhere vanishing on $\partial \Sigma$. Then $(\mathscr{M}, \mathfrak{g})$ is geodesically complete.

REMARK 4.2 The hypotheses of Theorem 4.1 are satisfied by all the metrics obtained by "Wick rotation" of metrics considered in the proof of Theorem 2.2 .

Proof: Along any maximally extended affinely parameterised geodesic $\Gamma(s)=$ $(t(s), \gamma(s))$ we have

$$
\frac{d}{d s}(\mathfrak{g}(X, \dot{\Gamma}))=0, \quad \mathfrak{g}(\dot{\Gamma}, \dot{\Gamma})=: \eta \in\{0, \pm 1\}
$$

Let $-\mathscr{E}$ denote $\mathfrak{g}(X, \dot{\Gamma})$, then $d \mathscr{E} / d s=0$ and

$$
\begin{aligned}
\frac{d t}{d s} & =\frac{\mathscr{E}}{V^{2}}, \\
g_{\Sigma}(\dot{\gamma}, \dot{\gamma}) & =\eta+\frac{\mathscr{E}^{2}}{V^{2}} .
\end{aligned}
$$

Since $V$ is strictly bounded away from zero, (4.3) implies that $t$ cannot blow up along $\Gamma$ in finite affine time $s$.

For spacelike geodesics (4.4) implies that $g_{\Sigma}(\dot{\gamma}, \dot{\gamma})$ is uniformly bounded, and completeness of $\Gamma$ follows from the geodesic completeness of $\left(\Sigma, g_{\Sigma}\right)$. 
For timelike geodesics we have $\eta=-1$ and since $g_{\Sigma}(\dot{\gamma}, \dot{\gamma}) \geq 0$, (4.4) shows that

$$
V^{2} \leq \mathscr{E}^{2}
$$

along $\Gamma$. It follows that $\Gamma$ stays away from the conformal boundary $\mathbb{R} \times \partial \Sigma$, and timelike geodesic completeness easily follows.

Null geodesics of $\mathfrak{g}$ are also null geodesics of the optical metric

$$
-d t^{2}+V^{-2} g_{\Sigma}
$$

The proof that any null geodesic which stays away from $\partial \Sigma$ is complete proceeds as in the timelike case. It remains to consider a null geodesic $\Gamma$ which approaches $\partial \Sigma$. Let $\hat{\Gamma}(r)=\Gamma(s(r))$ be a reparameterization of $\Gamma$ so that $r$ is an affine parameter with respect to the optical metric. Then $r$ can be chosen so that $\hat{\Gamma}(r)=(r, \hat{\gamma}(r))$, where $\hat{\gamma}$ is a unit-speed geodesic of the metric $\bar{g}_{\Sigma}$. By hypothesis $\hat{\gamma}$ meets $\partial \Sigma$ in finite time. The totally geodesic character of $\partial \Sigma$ implies that $\hat{\gamma}$ has to meet $\partial \Sigma$ transversally, and the completeness of $\Gamma$ follows from the usual formula for the change of affine parameter of null geodesics under conformal rescalings of the metric.

Returning to the justification of our claims from the introduction, we note the well known fact that ON-tetrad components of any finite number of covariant derivatives of the Riemann tensor are uniformly bounded on $M$ for all Riemannian conformally compactifiable metrics. In the obvious tetrad associated with the representation (3.4) of $g$, the algebraic invariants of the associated Lorentzian metric $\mathfrak{g}$ will be polynomials with constant coefficients of those tetrad components, and point 1 immediately follows. Global hyperbolicity in point 5 is established in the course of the proof of Theorem 4.1 of 13 .

It remains to consider the question of existence of other, perhaps only locally defined, Killing vector fields. Consider a space-time $(\mathscr{M}, \mathfrak{g})$ as in Theorem 4.1 and suppose that there exists an open subset of $\mathscr{M}$ with a Killing vector field $Y$ defined there, such that $Y$ is not proportional to $X$. Now, it is well known that $\Sigma$ admits an analytic atlas with respect to which $\mathfrak{g}$ is analytic. This allows us to make use of a theorem of Nomizu [31], and conclude that $Y$ can be extended to a globally defined Killing vector field on the universal cover $\mathbb{R} \times \hat{\Sigma}$ of $\mathbb{R} \times \Sigma$. In particular, for any point $p \in \partial \Sigma$ there exists a $\partial \Sigma$-neighborhood $\mathscr{U}$ of $p$ such that the algebra of Killing vector fields defined on $\mathbb{R} \times(\mathscr{U} \backslash \partial \Sigma)$ is at least two-dimensional. Now, Killing vector fields are conformal vector fields of the conformally rescaled metric. Further, conformal Killing vector fields satisfy an overdetermined system of ODE's of third order, which easily implies that they extend to the conformal boundary as conformal Killing vectors there. This can happen only for very special $\alpha$ 's and $g_{V}$ 's in (3.2), which justifies our claim 8 in the introduction. Point 7 is an obvious corollary of point 8 .

Identical arguments apply to those static solutions which have zeros of $u$, leading to black hole space-times.

ACKnowledgements: PTC acknowledges useful discussions with Witold Kondracki and Abdelghani Zeghib. 


\section{References}

[1] M.T. Anderson, On stationary vacuum solutions to the Einstein equations, Annales H. Poincaré 1 (2000), 977-994, gr-qc/0001091.

[2] - On the structure of solutions to the static vacuum Einstein equations, Annales H. Poincaré 1 (2000), 995-1042, gr-qc/0001018.

[3] _ Boundary regularity, uniqueness and non-uniqueness for AH Einstein metrics on 4-manifolds, Adv. in Math. (2001), in press, math.DG/0104171.

[4] _ Einstein metrics with prescribed conformal infinity on 4-manifolds, (2001), math.DG/0105243.

[5] L. Andersson and M. Dahl, Scalar curvature rigidity for asymptotically locally hyperbolic manifolds, Annals of Global Anal. and Geom. 16 (1998), $1-27$, dg-ga/9707017.

[6] J.J. van der Bij and E. Radu, New hairy black holes with negative cosmological constant, Phys. Lett. B536 (2002), 107-113, gr-qc/0107065.

[7] O. Biquard, Métriques d'Einstein asymptotiquement symétriques (Asymptotically symmetric Einstein metrics), Astérisque 265, Paris: Société Mathématique de France, 109 pp., 2000.

[8] J. Bjoraker and Y. Hosotani, Monopoles, dyons and black holes in the fourdimensional Einstein-Yang-Mills theory, Phys. Rev. D62 (2000), 043513.

[9] W. Boucher, G.W. Gibbons, and G.T. Horowitz, Uniqueness theorem for anti-de Sitter spacetime, Phys. Rev. D 30 (1984), 2447-2451.

[10] P.T. Chruściel, Uniqueness of black holes revisited, Helv. Phys. Acta 69 (1996), 529-552, Proceedings of Journés Relativistes 1996, Ascona, May 1996, N. Straumann,Ph. Jetzer and G. Lavrelashvili (Eds.), gr-qc/9610010.

[11] _ The classification of static vacuum space-times containing an asymptotically flat spacelike hypersurface with compact interior, Class. Quantum Grav. 16 (1999), 661-687, gr-qc/9809088.

[12] P.T. Chruściel and M. Herzlich, The mass of asymptotically hyperbolic Riemannian manifolds, (2001), dg-ga/0110035.

[13] P.T. Chruściel and W. Simon, Towards the classification of static vacuum spacetimes with negative cosmological constant, Jour. Math. Phys. 42 (2001), 1779-1817, gr-qc/0004032.

[14] R. Fintushel, Circle actions on simply connected 4-manifolds., Trans. Am. Math. Soc. 230 (1977), 147-171.

[15] Classification of circle actions on 4-manifolds., Trans. Am. Math. Soc. 242 (1978), 377-390. 
[16] G.J. Galloway, S. Surya, and E. Woolgar, On the geometry and mass of static, asymptotically AdS spacetimes, and the uniqueness of the AdS soliton, (2002), hep-th/0204081.

[17] _ A uniqueness theorem for the AdS soliton, Phys. Rev. Lett. 88 (2002), 101102, hep-th/0108170.

[18] G. W. Gibbons and S. W. Hawking, Classification of gravitational instanton symmetries, Commun. Math. Phys. 66 (1979), 291-310.

[19] C.R. Graham and J.M. Lee, Einstein metrics with prescribed conformal infinity on the ball, Adv. Math. 87 (1991), 186-225.

[20] S. W. Hawking and Don N. Page, Thermodynamics of black holes in anti-de sitter space, Commun. Math. Phys. 87 (1983), 577.

[21] M. Heusler, Black hole uniqueness theorems, Cambridge University Press, Cambridge, 1996.

[22] G.T. Horowitz and R.C. Myers, The AdS/CFT correspondence and a new positive energy conjecture for general relativity, Phys. Rev. D59 (1999), 026005 (12 pp.).

[23] E. Kasner, Solutions of the Einstein equations involving functions of only one variable, Trans. AMS 27 (1925), 155-162.

[24] S. Kobayashi, Transformation groups in differential geometry, Ergebnisse der Math., vol. 70, Springer-Verlag, New York, 1972.

[25] D. Korotkin and H. Nicolai, A periodic analog of the Schwarzschild solution, (1994), gr-qc/9403029.

[26] D. Kramer, H. Stephani, M. MacCallum, and E. Herlt, Exact solutions of Einstein's field equations, Cambridge University Press, Cambridge, 1980.

[27] J. Lafontaine and L. Rozoy, (2002), in preparation.

[28] J. Lee, Fredholm operators and Einstein metrics on conformally compact manifolds, math.DG/0105046, 2001.

[29] B. Linet, The static, cylindrically symmetric strings in general relativity with cosmological constant, Jour. Math. Phys. 27 (1986), 1817-1818.

[30] Robert C. Myers, Higher dimensional black holes in compactified spacetimes, Phys. Rev. D35 (1987), 455-466.

[31] K. Nomizu, On local and global existence of Killing vector fields, Ann. Math. 72 (1960), 105-120.

[32] S. Smale, An infinite dimensional version of Sard's theorem, Am. Jour. Math. 87 (1965), 861-866. 
[33] G. Weinstein, N-black hole stationary and axially symmetric solutions of the Einstein/Maxwell equations, Commun. Part. Diff. Eqs. 21 (1996), 1389-1430.

[34] E. Winstanley and O. Sarbach, On the linear stability of solitons and hairy black holes with a negative cosmological constant: The even-parity sector, Class. Quant. Grav. 19 (2002), 689-724. 